\title{
Net ultrafiltration intensity and mortality in critically ill patients with fluid overload
}

Raghavan Murugan ${ }^{1,2,7^{*}}$ (D), Vikram Balakumar ${ }^{1,2}$, Samantha J. Kerti ${ }^{2}$, Priyanka Priyanka ${ }^{1,2}$, Chung-Chou H. Chang ${ }^{1,2,3,4}$, Gilles Clermont ${ }^{2}$, Rinaldo Bellomo5, Paul M. Palevsky ${ }^{1,3,6}$ and John A. Kellum,2

\begin{abstract}
Background: Although net ultrafiltration $\left(\mathrm{UF}^{\mathrm{NET}}\right)$ is frequently used for treatment of fluid overload in critically ill patients with acute kidney injury, the optimal intensity of UF ${ }^{\mathrm{NET}}$ is unclear. Among critically ill patients with fluid overload receiving renal replacement therapy (RRT), we examined the association between UF ${ }^{\mathrm{NET}}$ intensity and riskadjusted 1-year mortality.

Methods: We selected patients with fluid overload $\geq 5 \%$ of body weight prior to initiation of RRT from a large academic medical center ICU dataset. UF ${ }^{\mathrm{NET}}$ intensity was calculated as the net volume of fluid ultrafiltered per day from initiation of either continuous or intermittent RRT until the end of ICU stay adjusted for patient hospital admission body weight. We stratified UF ${ }^{\mathrm{NET}}$ as low $(\leq 20 \mathrm{ml} / \mathrm{kg} /$ day), moderate $(>20$ to $\leq 25 \mathrm{ml} / \mathrm{kg} /$ day) or high (> $25 \mathrm{ml} / \mathrm{kg} /$ day) intensity. We adjusted for age, sex, body mass index, race, surgery, baseline estimated glomerular filtration rate, oliguria, first RRT modality, pre-RRT fluid balance, duration of RRT, time to RRT initiation from ICU admission, APACHE III score, mechanical ventilation use, suspected sepsis, mean arterial pressure on day 1 of RRT, cumulative fluid balance during RRT and cumulative vasopressor dose during RRT. We fitted logistic regression for 1-year mortality, Gray's survival model and propensity matching to account for indication bias.
\end{abstract}

Results: Of 1075 patients, the distribution of high, moderate and low-intensity UF ${ }^{\mathrm{NET}}$ groups was $40.4 \%, 15.2 \%$ and $44.2 \%$ and 1 -year mortality was $59.4 \%$ vs $60.2 \%$ vs $69.7 \%$, respectively $(p=0.003)$. Using logistic regression, highintensity compared with low-intensity UF ${ }^{\mathrm{NET}}$ was associated with lower mortality (adjusted odds ratio $0.61,95 \% \mathrm{Cl}$ $0.41-0.93, p=0.02$ ). Using Gray's model, high UF ${ }^{\mathrm{NET}}$ was associated with decreased mortality up to 39 days after ICU admission (adjusted hazard ratio range $0.50-0.73$ ). After combining low and moderate-intensity UF ${ }^{\mathrm{NET}}$ groups $(n=258)$ and propensity matching with the high-intensity group $(n=258), \mathrm{UF}^{\mathrm{NET}}$ intensity $>25 \mathrm{ml} / \mathrm{kg} /$ day compared with $\leq$ $25 \mathrm{ml} / \mathrm{kg} /$ day was associated with lower mortality ( $57 \%$ vs $67.8 \%, p=0.01$ ). Findings were robust to several sensitivity analyses.

Conclusions: Among critically ill patients with $\geq 5 \%$ fluid overload and receiving RRT, UF ${ }^{\text {NET }}$ intensity $>25 \mathrm{ml} / \mathrm{kg} /$ day compared with $\leq 20 \mathrm{ml} / \mathrm{kg} /$ day was associated with lower 1 -year risk-adjusted mortality. Whether tolerating intensive $U \mathrm{~F}^{\mathrm{NET}}$ is just a marker for recovery or a mediator requires further research.

Keywords: Net ultrafiltration, Intensity, Fluid overload, Renal replacement therapy, Dialysis, Mortality

\footnotetext{
* Correspondence: muruganr@upmc.edu

'Department of Critical Care Medicine, The Center for Critical Care

Nephrology, CRISMA, University of Pittsburgh School of Medicine, Pittsburgh,

PA, USA

${ }^{2}$ Department of Critical Care Medicine, The Clinical Research, Investigation,

and Systems Modeling of Acute IIIness (CRISMA) Center, University of

Pittsburgh School of Medicine, Pittsburgh, PA, USA

Full list of author information is available at the end of the article
}

(c) The Author(s). 2018 Open Access This article is distributed under the terms of the Creative Commons Attribution 4.0 International License (http://creativecommons.org/licenses/by/4.0/), which permits unrestricted use, distribution, and reproduction in any medium, provided you give appropriate credit to the original author(s) and the source, provide a link to the Creative Commons license, and indicate if changes were made. The Creative Commons Public Domain Dedication waiver (http://creativecommons.org/publicdomain/zero/1.0/) applies to the data made available in this article, unless otherwise stated. 


\section{Background}

Fluid overload (FO) is a common complication of acute illness affecting more than a third of critically ill patients and approximately two-thirds of patients with acute kidney injury (AKI) requiring renal replacement therapy (RRT) $[1,2]$. Several studies have documented that FO is independently associated with more than $50 \%$ mortality among patients receiving RRT [3, 4]. Observational studies suggest that fluid removal using net ultrafiltration $\left(\mathrm{UF}^{\mathrm{NET}}\right)$ may be associated with improved outcomes [2], and clinical and consensus guidelines recommend $\mathrm{UF}^{\mathrm{NET}}$ for the treatment of FO in patients with oliguric AKI who are resistant to diuretic therapy $[5,6]$. However, the optimal intensity of $\mathrm{UF}^{\mathrm{NET}}$ (i.e., rate and volume of net fluid removal) in critically ill patients remains uncertain more than 70 years after the first clinical use of ultrafiltration [7].

Less intensive $\mathrm{UF}^{\mathrm{NET}}$, characterized by a slower rate or smaller volume of fluid removed, may be associated with prolonged exposure to tissue and organ edema and increased morbidity and mortality [8,9]. More intensive $\mathrm{UF}^{\mathrm{NET}}$ with a faster rate or larger volume of fluid removal, however, may be associated with increased hemodynamic and cardiovascular stress [10], leading to ischemic organ injury and mortality in critically ill patients [11]. Indeed, three observational studies in outpatients with end-stage renal disease suggest that UF ${ }^{\mathrm{NET}}$ intensity $>10 \mathrm{ml} / \mathrm{kg} / \mathrm{h}$ is associated with increased overall [12-14] and cardiovascular [12] mortality.

Understanding the relationship between $\mathrm{UF}^{\mathrm{NET}}$ intensity and outcome in critically ill patients is essential for two important reasons. First, if more intensive $U^{\mathrm{NET}}$ is associated with lower mortality, then clinical trials could be designed to reduce the risk of death. Second, understanding the intensity-outcome relationship will aid in standardizing $\mathrm{UF}^{\mathrm{NET}}$ intensity and implementing quality measures $[15,16]$.

In this observational study involving a large heterogeneous cohort of critically ill patients with $\geq 5 \%$ FO and receiving RRT, we examined the association between $\mathrm{UF}^{\mathrm{NET}}$ intensity and its association with risk-adjusted 1 -year mortality. Because the magnitude of FO is independently associated with mortality, we hypothesized that intensive $\mathrm{UF}^{\mathrm{NET}}$ would be associated with lower mortality. However, our null hypothesis was that there is no difference in mortality for an intensive $\mathrm{UF}^{\mathrm{NET}}$ group compared with a less intensive $\mathrm{UF}^{\mathrm{NET}}$ group.

\section{Methods}

\section{Data source and study population}

We conducted a retrospective study using a large tertiary care academic medical center ICU database: the High-Density Intensive Care dataset, details of which have been published elsewhere (Additional file 1: S1) [1,
17, 18]. The study population included adults admitted to medical, cardiac, abdominal transplant, cardiothoracic, surgical, neurovascular, neurotrauma and trauma ICUs during July 2000 through October 2008. We included patients with AKI receiving RRT who had a cumulative fluid balance $\geq 5 \%$ prior to RRT initiation (Additional file 1: Figure S1). We extracted the daily fluid balance before and for the duration of RRT (Additional file 1: S2), hourly mean arterial pressure (MAP) and vasopressor type and dose (Additional file 1: S3) during RRT. The University of Pittsburgh's institutional review board approved the study.

\section{Determination of UF ${ }^{\mathrm{NET}}$ intensity}

For patients receiving continuous renal replacement therapy (CRRT), we first extracted data on the total duration (in hours) of any form of CRRT (i.e., continuous venovenous hemodiafiltration (CVVHDF), continuous venovenous hemofiltration $(\mathrm{CVVH})$, continuous venovenous hemodialysis (CVVHD) and slow continuous ultrafiltration (SCUF)). We then determined the UF volume produced and the amount of substitution fluids given each hour for patients receiving CVVHDF and CVVH. The UF ${ }^{\mathrm{NET}}$ each hour was calculated as the difference between the UF volume and the volume of substitution fluids [19]. For patients receiving CVVHD and SCUF, $\mathrm{UF}^{\mathrm{NET}}$ corresponded to the UF volume removed. We then calculated the total number of days of CRRT for each patient based on the hourly duration of CRRT and the total UF ${ }^{\mathrm{NET}}$.

For patients receiving intermittent hemodialysis (IHD), we extracted the total number of IHD sessions and the UF volume removed per session from the time of ICU admission to the end of ICU stay. We excluded patients if they received IHD prior to ICU admission. $\mathrm{UF}^{\mathrm{NET}}$ corresponded to the volume ultrafiltered during each session. We then expressed the total number of IHD sessions as the number of days for each patient. Subsequently, we estimated the UF ${ }^{\mathrm{NET}}$ intensity using the equation:

$$
\begin{aligned}
& \mathrm{UF}^{\mathrm{NET}} \text { intensity }(\mathrm{ml} / \mathrm{kg} / \text { day }) \\
& =\frac{\text { Total } \mathrm{UF}^{\mathrm{NET}} \text { volume }(\mathrm{ml})}{\text { Hospital admission weight }(\mathrm{kg}) \mathrm{X} \text { RRT duration (days) }} .
\end{aligned}
$$

For instance, if an $80-\mathrm{kg}$ patient is on CVVH with an UF rate of $2000 \mathrm{ml} / \mathrm{h}$ and substitution fluid of $1500 \mathrm{ml} /$ $\mathrm{h}$, the total $\mathrm{UF}^{\mathrm{NET}}$ produced is $500 \mathrm{ml} / \mathrm{h}(2000-1500=$ $500 \mathrm{ml}$ ) or $500 \times 24=12,000 \mathrm{ml} /$ day. The total $\mathrm{UF}^{\mathrm{NET}}$ produced for 5 days is $12,000 \times 5=60,000 \mathrm{ml}$. Thus, the total $\mathrm{UF}^{\mathrm{NET}}$ intensity is $[60,000 /(80 \times 5)]=150 \mathrm{ml} / \mathrm{kg} /$ day. During CVVHD and IHD, the UF volume is equivalent to $\mathrm{UF}^{\mathrm{NET}}$. 


\section{Outcomes}

The primary outcome was 1-year mortality from the index ICU admission and mortality data were obtained from the Social Security Death Master File [20]. We chose 1-year mortality because our prior work showed that a positive fluid balance was associated with risk of death at 1 year and use of renal replacement therapy was associated with lower risk of death in patients with a positive fluid balance [1]. Secondary outcomes included hospital length of stay, hospital mortality and renal recovery. Renal recovery was defined as alive and independent from RRT at 1 year. Dialysis dependence data were obtained from the US Renal Data System [21].

\section{Statistical analysis}

We stratified UF ${ }^{\mathrm{NET}}$ intensity into three groups because of the nonlinear (i.e., J-shaped) association between $\mathrm{UF}^{\mathrm{NET}}$ intensity and hospital mortality (Additional file 1: Figure S2). We defined $\mathrm{UF}^{\mathrm{NET}} \leq 20 \mathrm{ml} / \mathrm{kg} /$ day as "low" intensity, UF ${ }^{\mathrm{NET}}>20$ to $\leq 25 \mathrm{ml} / \mathrm{kg} /$ day as "moderate" intensity and $\mathrm{UF}^{\mathrm{NET}}>25 \mathrm{ml} / \mathrm{kg} /$ day as "high" intensity. Categorical variables were compared using the chisquared test, and continuous variables using-one way analysis of variance and the Kruskal-Wallis test. We assessed time-to-mortality censored at 1 year using Kaplan-Meier failure plots.

We used three methods to examine the association between $\mathrm{UF}^{\mathrm{NET}}$ intensity and mortality. First, we fitted logistic regression and estimated risk-adjusted odds ratios (AORs) for high and moderate intensity, compared with low intensity $\mathrm{UF}^{\mathrm{NET}}$ (reference), on 1-year mortality. Second, we fitted Gray's survival model $[22,23]$ to estimate risk-adjusted hazard ratios (AHRs) for time to mortality using four time nodes and five intervals (Additional file 1: S4). We adjusted for differences in age, sex, race, body mass index, history of liver disease and sequela from liver disease, admission for liver transplantation, admission for surgery, baseline glomerular filtration rate, Acute Physiologic and Chronic Health Evaluation (APACHE) III score, presence of sepsis, use of mechanical ventilation, percentage of FO before initiation of RRT, oliguria before initiation of RRT, time to initiation of RRT from ICU admission, MAP on first day of RRT initiation, cumulative vasopressor dose and cumulative fluid balance during RRT, first RRT modality and duration of RRT.

Third, in order to account for indication bias, we conducted a propensity score-matched analysis. Since the mortality associated with moderate ( $>20$ to $\leq 25 \mathrm{ml} / \mathrm{kg}$ / day) vs high (>25 ml/kg/day) or moderate (> 20 to $\leq$ $25 \mathrm{ml} / \mathrm{kg} /$ day $)$ vs low ( $\leq 20 \mathrm{ml} / \mathrm{kg} /$ day $)$ intensity $\mathrm{UF}^{\mathrm{NET}}$ was not different (Table 1), we combined the moderate and low-intensity groups into a single low-intensity group (reference). We then matched the low-intensity $\mathrm{UF}^{\mathrm{NET}}$ ( $\leq 25 \mathrm{ml} / \mathrm{kg} /$ day) with the high-intensity $\mathrm{UF}^{\mathrm{NET}}$
(> $25 \mathrm{ml} / \mathrm{kg} /$ day) using propensity scores on a 1:1 basis without replacement, creating 258 matched pairs (Additional file 1: S5).

We performed five sensitivity analyses and two subgroup analyses. First, we restricted the UF ${ }^{\mathrm{NET}}$ intensity only up to $72 \mathrm{~h}$ from initiation of RRT. Second, we used an alternative definition of $\mathrm{UF}^{\mathrm{NET}}$ intensity moving the threshold down as follows: low, $<15 \mathrm{ml} / \mathrm{kg} /$ day; moderate, $15-20 \mathrm{ml} / \mathrm{kg} /$ day; and high, $>20 \mathrm{ml} / \mathrm{kg} /$ day. Third, we moved the threshold up: low, $<25 \mathrm{ml} / \mathrm{kg} /$ day; moderate, $25-30 \mathrm{ml} / \mathrm{kg} /$ day; and high, $>30 \mathrm{ml} / \mathrm{kg} /$ day. Fourth, we divided the cohort into tertiles: low, $\leq 16.7 \mathrm{ml} / \mathrm{kg} /$ day; moderate, 16.7 to $\leq 27.7 \mathrm{ml} / \mathrm{kg} /$ day; and high, > $27.7 \mathrm{ml} / \mathrm{kg} /$ day. Fifth, we performed quantitative bias analysis to assess the magnitude of a hypothetical unmeasured confounder that would be necessary to account for the association between $\mathrm{UF}^{\mathrm{NET}}$ intensity and risk-adjusted mortality (Additional file 1: S6) [24, 25].

Sixth, we restricted our analyses only to the subgroup of patients with $>20 \%$ FO. Seventh, we confined our analysis of UF ${ }^{\mathrm{NET}}$ intensity to the hour (i.e., $\mathrm{ml} / \mathrm{kg} / \mathrm{h}$ ) instead of the day among the subgroup of patients who only received CRRT as follows: low, $<0.5 \mathrm{ml} / \mathrm{kg} / \mathrm{h}$; $\bmod$ erate, $0.5-1.0 \mathrm{ml} / \mathrm{kg} / \mathrm{h}$; and high, $>1 \mathrm{ml} / \mathrm{kg} / \mathrm{h}$. Statistical analyses were performed using SAS 9.3 (SAS Institute, Cary, NC, USA), Gray's model used R 3.2.1, and quantitative bias analysis was performed using STATA 15 (STATCorp., TX, USA). All hypotheses tests were twosided with a significance level of $p<0.05$.

\section{Results}

\section{Study population and patient characteristics}

Of 45,568 patients, we excluded patients with no available baseline weight $(n=2214)$, ICU duration $\leq 48 \mathrm{~h} \quad(n=$ $18,032)$, death within $72 \mathrm{~h}$ of ICU admission $(n=663)$, chronic dialysis $(n=2386)$, admission for or with history of renal transplantation $(n=1232)$, serum creatinine $\geq 3.5$ $\mathrm{mg} / \mathrm{dl}$ within 1 year of hospitalization $(n=147)$ and missing data on fluid balance $(n=2810)$. Of 18,084 patients in whom cumulative fluid balance data were available, we excluded those with cumulative fluid balance $<5 \%$ of body weight $(n=9900)$. Of patients with cumulative balance $\geq$ $5 \%$ of body weight $(n=8184)$, we excluded those who did not receive RRT $(n=7023)$ and patients without data on $\mathrm{UF}^{\mathrm{NET}}(n=86)$ (Additional file 1: Figure S1).

Of 1075 patients, the distribution of low, moderate and high-intensity $\mathrm{UF}^{\mathrm{NET}}$ groups was $44.2 \%, 15.2 \%$ and $40.4 \%$, respectively. Minor differences were noted among male sex, race and body mass index between the groups (Table 1). There was a higher prevalence of liver disease (34.5\%), sequela from liver disease $(28.8 \%)$ and liver transplantation $(21.5 \%)$ among those with low-intensity $\mathrm{UF}^{\mathrm{NET}}$. There was a higher prevalence of oliguria in those who received moderate and high-intensity $\mathrm{UF}^{\mathrm{NET}}$. 
Table 1 Baseline characteristics of study population by net ultrafiltration Intensity

\begin{tabular}{|c|c|c|c|c|}
\hline & $\leq 20 \mathrm{ml} / \mathrm{kg} /$ day $(n=475)$ & $>20$ to $\leq 25 \mathrm{ml} / \mathrm{kg} /$ day $(n=166)$ & $>25 \mathrm{ml} / \mathrm{kg} /$ day $(n=434)$ & $p$ value \\
\hline Age (years), median (IQR) & $61(52-69)$ & $59(51-71)$ & $58(48-70)$ & 0.16 \\
\hline Male sex & $301(63.4)$ & $114(68.7)$ & $218(50.2)$ & $<0.001$ \\
\hline \multicolumn{5}{|l|}{ Race } \\
\hline Caucasian & $380(80)$ & $136(81.9)$ & $335(77.2)$ & \multirow[t]{3}{*}{0.018} \\
\hline African-American & $24(5.1)$ & $6(3.6)$ & $43(9.9)$ & \\
\hline Other & $71(14.9)$ & $24(14.5)$ & $56(12.9)$ & \\
\hline BMI $\left(\mathrm{kg} / \mathrm{m}^{2}\right)$, median (IQR) & $28.3(24.2-34.3)$ & $27.7(24.2-31.7)$ & $25.1(21.9-29.3)$ & $<0.001$ \\
\hline \multicolumn{5}{|l|}{ Comorbid condition } \\
\hline Hypertension & 169 (35.6) & $72(43.4)$ & $161(37.1)$ & 0.19 \\
\hline Diabetes & $121(25.5)$ & $34(20.5)$ & $97(22.4)$ & 0.33 \\
\hline Cardiac disease & $84(17.7)$ & $36(21.7)$ & $99(22.8)$ & 0.14 \\
\hline Heart failure & $70(14.7)$ & $30(18.1)$ & $86(19.8)$ & 0.12 \\
\hline Vascular disease & $41(8.6)$ & $16(9.6)$ & $43(9.9)$ & 0.79 \\
\hline Liver disease & $164(34.5)$ & $47(28.3)$ & $107(24.7)$ & 0.005 \\
\hline Sequela from liver disease & $137(28.8)$ & $43(25.9)$ & $95(21.9)$ & 0.056 \\
\hline Malignancy & $23(4.8)$ & $4(2.4)$ & $14(3.2)$ & 0.26 \\
\hline Liver transplantation & $43(9.1)$ & $13(7.8)$ & $42(9.7)$ & 0.77 \\
\hline Multiple comorbidity & $298(62.7)$ & $93(56)$ & $252(58.1)$ & 0.19 \\
\hline Surgical admission & $321(67.6)$ & $122(73.5)$ & $301(69.4)$ & 0.72 \\
\hline Medical admission & $131(27.6)$ & $37(22.3)$ & $112(25.8)$ & 0.72 \\
\hline Admission for liver transplantation & $102(21.5)$ & $31(18.7)$ & $53(12.2)$ & 0.001 \\
\hline $\begin{array}{l}\text { Baseline serum creatinine }(\mathrm{mg} / \mathrm{dl}) \text {, } \\
\text { median (IQR) }\end{array}$ & $1.029(0.81-1.27)$ & $1.035(0.83-1.3)$ & $1.032(0.8-1.3)$ & 0.89 \\
\hline \multicolumn{5}{|l|}{ Baseline eGFR (ml/min/1.73 $\left.\mathrm{m}^{2}\right)$} \\
\hline$>90$ & $107(22.5)$ & $27(16.3)$ & $91(20.9)$ & \multirow[t]{5}{*}{0.54} \\
\hline $60-90$ & $235(49.5)$ & $97(58.4)$ & $212(48.9)$ & \\
\hline $30-60$ & 89 (18.7) & $30(18.1)$ & $92(21.2)$ & \\
\hline $15-30$ & $34(7.2)$ & $8(4.8)$ & $31(7.1)$ & \\
\hline$<15$ & $10(2.1)$ & $4(2.4)$ & $8(1.8)$ & \\
\hline APACHE III score, median (IQR) ${ }^{a}$ & $95(70-118)$ & $91(71-116)$ & $91(69-112)$ & 0.27 \\
\hline Sepsis ${ }^{a}$ & $128(26.9)$ & $39(23.5)$ & $138(31.8)$ & 0.08 \\
\hline Mechanical ventilation ${ }^{a}$ & $353(74.3)$ & $129(77.7)$ & $329(75.8)$ & 0.66 \\
\hline Vasopressor $^{\mathrm{a}}$ & $261(54.9)$ & $87(52.4)$ & $218(50.2)$ & 0.36 \\
\hline \multicolumn{5}{|l|}{ Oliguria before initiation of RRT ${ }^{\mathrm{b}}$} \\
\hline Stage 2 & $50(10.5)$ & $9(5.4)$ & $21(4.8)$ & \multirow[t]{2}{*}{0.017} \\
\hline Stage 3 & $406(85.5)$ & $154(92.8)$ & $402(92.6)$ & \\
\hline \multicolumn{5}{|l|}{ MAP during RRT (mmHg), mean (SD) } \\
\hline All patients & $75.1(0.58)$ & $77.5(1.19)$ & $79.4(0.62)$ & $<0.001$ \\
\hline CRRT only $(n=386)$ & $72.7(0.70)$ & $72.4(1.89)$ & $77.5(1.01)$ & $<0.001$ \\
\hline IHD only $(n=210)$ & $85(1.84)$ & $84.1(2.85)$ & $82.1(1.27)$ & 0.77 \\
\hline CRRT and IHD $(n=487)$ & $74.5(0.91)$ & $79.1(1.66)$ & $79.7(0.98)$ & 0.002 \\
\hline \multicolumn{5}{|l|}{ Vasopressor dose (NE), median (IQR) ${ }^{\mathrm{c}, \mathrm{d}}$} \\
\hline All patients & $0.11(0.04-0.25)$ & $0.09(0.03-0.21)$ & $0.09(0.04-0.25)$ & 0.25 \\
\hline Patients on CRRT only & $0.14(0.05-0.30)$ & $0.13(0.03-0.25)$ & $0.10(0.03-0.28)$ & 0.31 \\
\hline
\end{tabular}


Table 1 Baseline characteristics of study population by net ultrafiltration Intensity (Continued)

\begin{tabular}{lllll}
\hline & $\leq 20 \mathrm{ml} / \mathrm{kg} / \mathrm{day}(n=475)$ & $>20$ to $\leq 25 \mathrm{ml} / \mathrm{kg} /$ day $(n=166)$ & $>25 \mathrm{ml} / \mathrm{kg} / \mathrm{day}(n=434)$ & $p$ value \\
\hline Patients on IHD only & $0.01(0.01-0.03)$ & $0.06(0.01-0.11)$ & $0.03(0.01-0.07)$ & 0.67 \\
Patients on both CRRT and IHD & $0.08(0.03-0.16)$ & $0.08(0.02-0.16)$ & $0.07(0.03-0.19)$ & 0.85 \\
\hline
\end{tabular}

Data presented as $n(\%)$ unless stated otherwise

$I Q R$ interquartile range, $B M I$ body mass index, eGFR estimated glomerular filtration, APACHE Acute Physiology and Chronic Health Evaluation, RRT renal replacement therapy, MAP mean arterial pressure, $S D$ standard deviation, $C R R T$ continuous renal replacement therapy, IHD intermittent hemodialysis, $N E$ norepinephrine equivalents

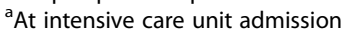

${ }^{b}$ Patients were classified to have developed oliguria according to the maximum Kidney Disease Improving Global Outcome criteria based on urine output [5] 'On the day 1 of RRT

${ }^{d}$ All vasopressors were standardized in terms of NE (Additional file 1: S3) [30-32]

Patients in the low-intensity $\mathrm{UF}^{\mathrm{NET}}$ group had lower MAP compared with the moderate and high-intensity $\mathrm{UF}^{\mathrm{NET}}$ groups (Table 1 and Additional file 1: Table S1).

Cumulative FO before RRT initiation was lowest in the low-intensity group, compared with the moderate and high-intensity $\mathrm{UF}^{\mathrm{NET}}$ groups $(15.6 \%$ vs $17.3 \%$ vs $21 \%$ of body weight, respectively, $p<0.001$; Table 2). Following initiation of RRT, the median cumulative FB for the low, medium and high-intensity $\mathrm{UF}^{\mathrm{NET}}$ groups was 13.5 vs 22 vs $191, p<0.001$; Table 2). During RRT, the MAP was lower and the cumulative vasopressor dose was higher in the low-intensity $\mathrm{UF}^{\mathrm{NET}}$ group compared with the moderate and high-intensity $\mathrm{UF}^{\mathrm{NET}}$ groups (Table 2 and Additional file 1: Table S1).

The median duration of RRT for the low, moderate and high-intensity $\mathrm{UF}^{\mathrm{NET}}$ groups was 4.7 vs 8.7 vs 7 days, respectively $(p<0.001)$. The median duration of CRRT was 3.9 vs 5.8 vs 5.9 days $(p<0.001)$ and the median $\mathrm{UF}^{\mathrm{NET}}$ volume was 3.4 vs 11.6 vs $16.2 \mathrm{~L}(p<0.001)$. The median duration of IHD was 2 vs 7 vs 4 days $(p=0.004)$ and the median $\mathrm{UF}^{\mathrm{NET}}$ volume was 5.5 vs 12.6 vs $9.2 \mathrm{~L}(p<0.001)$. The median duration of RRT for patients who received both CRRT and IHD was 14.7 vs 15.2 vs 10.7 days ( $p<$ 0.001 ) and the median $U^{\mathrm{NET}}$ volume was 19.5 vs 27.9 vs $26.6 \mathrm{~L}(p<0.001)$. The median hospital length of stay was 32 vs 37.5 vs 37 days $(p<0.001)$ (Table 2$)$. This shorter length of stay among patients with low-intensity $\mathrm{UF}^{\mathrm{NET}}$ was primarily due to higher mortality in this group. However, there was no difference in renal recovery at 1 year ( $25.1 \%$ vs $28.9 \%$ vs $31.8 \%, p=0.078$ ) as well as within the subgroup of survivors at 1 year $(82.6 \%$ vs $72.7 \%$ vs $78.4 \%$, $p=0.25$ ) between the three groups.

\section{Association between UF ${ }^{\mathrm{NET}}$ intensity and mortality}

The crude hospital and 1-year mortality was higher among the low-intensity group compared with the moderate and high-intensity UF ${ }^{\mathrm{NET}}$ groups: $69.7 \%$ vs $60.2 \%$ vs $59.4 \%$ ( $p=$ 0.003 ), respectively (Table 2, Fig. 1a). Using logistic regression, high-intensity compared with low-intensity $\mathrm{UF}^{\mathrm{NET}}$ was associated with lower 1-year mortality (AOR 0.61, 95\% CI $0.41-0.93, p=0.02, C$-statistic 0.811; Table 3 and Additional file 1: Table S2). This association persisted using
$\mathrm{UF}^{\mathrm{NET}}$ as a continuous variable (AOR $0.98,95 \%$ CI $0.97-$ $0.99, p=0.005$; Additional file 1: Table S3). Compared with $\mathrm{UF}^{\mathrm{NET}}$ of $0-5 \mathrm{ml} / \mathrm{kg} /$ day, increasing $\mathrm{UF}^{\mathrm{NET}}$ intensity was associated with a trend toward lower odds of death $(C$-statistic - 0.813; Fig. 1b), whereas moderate-intensity compared with low-intensity $\mathrm{UF}^{\mathrm{NET}}$ was not associated with mortality (AOR 0.81, 95\% CI 0.48-1.35, $p=0.41$; Additional file 1: Table S2).

Using Gray's model, high-intensity compared with low-intensity $\mathrm{UF}^{\mathrm{NET}}$ had variable association with mortality. Early on after ICU admission, high-intensity UF ${ }^{\mathrm{NET}}$ was associated with lowest risk of death that was subsequently attenuated over time, but nevertheless persisted up to 39 days after ICU admission (AHR range 0.50-0.73, $p<0.001$; Table 4 and Additional file 1: Figure S3A). Subsequently, between 39 and 365 days, high-intensity $\mathrm{UF}^{\mathrm{NET}}$ was not associated with mortality (AHR range 0.76-1.02). High-intensity compared with moderate-intensity $\mathrm{UF}^{\mathrm{NET}}$ was only associated with lower risk of death up to 15 days (AHR 0.53, 95\% CI 0.33-0.86; Additional file 1: Table S5 and Figure S3C).

After propensity matching, 258 matched pairs were created wherein patients with $\mathrm{UF}^{\mathrm{NET}}$ intensity $\leq 25 \mathrm{ml} / \mathrm{kg} /$ day had similar baseline characteristics compared with $\mathrm{UF}^{\mathrm{NET}}$ intensity $>25 \mathrm{ml} / \mathrm{kg} /$ day, except for cumulative vasopressor dose (Additional file 1: Table S4). Patients with $\mathrm{UF}^{\mathrm{NET}}$ intensity $>25 \mathrm{ml} / \mathrm{kg} /$ day compared with $\leq$ $25 \mathrm{ml} / \mathrm{kg} /$ day had lower 1 -year mortality (57\% vs $67.8 \%, p$ $=0.01$; Fig. 2), which persisted after adjusting for vasopressor dose (AOR 0.63, 95\% CI 0.44-0.90, $p=0.011$ ).

\section{Sensitivity analyses}

When $\mathrm{UF}^{\mathrm{NET}}$ intensity calculation was limited within $72 \mathrm{~h}$ of initiation of RRT, high-intensity $\mathrm{UF}^{\mathrm{NET}}$ was associated with lower mortality (AOR 0.56 , 95\% CI $0.35-$ $0.88, p=0.013$; Table 5). Using the alternative thresholds of low, moderate and high-intensity $\mathrm{UF}^{\mathrm{NET}}$ of $<15 \mathrm{ml} /$ $\mathrm{kg} / \mathrm{h}, 15-20 \mathrm{ml} / \mathrm{kg} / \mathrm{h}$ and $>20 \mathrm{ml} / \mathrm{kg} / \mathrm{h}$, respectively, we found $\mathrm{UF}^{\mathrm{NET}}$ intensity $>20 \mathrm{ml} / \mathrm{kg} / \mathrm{h}$ was associated with lower mortality (AOR 0.63, 95\% CI 0.41-0.97, $p=0.038$ ). Similar results were found moving the threshold up (AOR 0.58, 95\% CI 0.34-0.99, $p=0.04$; Table 5) and 
Table 2 Fluid balance, RRT characteristics and outcomes by intensity of net ultrafiltration

\begin{tabular}{|c|c|c|c|c|}
\hline & $\begin{array}{l}\leq 20 \\
\mathrm{ml} / \mathrm{kg} / \mathrm{day} \\
(n=475)\end{array}$ & $\begin{array}{l}>20 \text { to } \leq 25 \\
\mathrm{ml} / \mathrm{kg} / \mathrm{day} \\
(n=166)\end{array}$ & $\begin{array}{l}>25 \\
\mathrm{ml} / \mathrm{kg} / \text { day } \\
(n=434)\end{array}$ & $p$ value \\
\hline $\begin{array}{l}\text { Fluids administered in the first } 24 \mathrm{~h} \text { of ICU admission (L), } \\
\text { median (IQR) }\end{array}$ & $5.3(3.5-7.9)$ & $5.1(3.6-7.8)$ & $5.23(3.3-8.1)$ & 0.88 \\
\hline \multicolumn{5}{|l|}{ Fluid balance after ICU admission (L), median (IQR) } \\
\hline At $72 \mathrm{~h}$ & $7.9(4.4-12)$ & $7.8(4.7-13.3)$ & $7.6(4.7-11.6)$ & 0.71 \\
\hline At 7 days & $10.1(6.7-15.2)$ & $10.5(6.4-15.7)$ & $10.1(6.4-15.1)$ & 0.78 \\
\hline Average before RRT & $2.3(1.2-4.4)$ & $2.7(1.5-4.3)$ & $2.3(1.2-4.2)$ & 0.33 \\
\hline Cumulative before RRT (\%) & $15.6(10-25)$ & $17.3(9.9-28.6)$ & $21(12.4-33.7)$ & $<0.001$ \\
\hline Duration from ICU admission to RRT (days), median (IQR) & $7(2-13)$ & $5(3-12)$ & $6(3-16)$ & 0.27 \\
\hline RRT duration (days), median (IQR) & $4.7(1.5-11.7)$ & $8.7(4.5-16.7)$ & $7(3.1-12.7)$ & $<0.001$ \\
\hline $\begin{array}{l}\text { Cumulative FB excluding UF }{ }^{\mathrm{NET}} \text { for duration of RRT }(\mathrm{L}) \text {, } \\
\text { median (IQR) }\end{array}$ & $13.5(4.2-32.8)$ & $22(8.9-45.1)$ & $19(7.3-37.2)$ & $<0.001$ \\
\hline MAP for duration of RRT (mmHg), mean (SD) & $75.2(0.6)$ & $77.4(0.8)$ & $80.1(0.53)$ & $<0.001$ \\
\hline $\begin{array}{l}\text { Cumulative vasopressor dose for duration of RRT (NE), } \\
\text { median (IQR) }\end{array}$ & $15.7(4.3-38.6)$ & $11.4(1.2-34.7)$ & $8.1(0.9-25.7)$ & $<0.001$ \\
\hline \multicolumn{5}{|l|}{ First RRT modality } \\
\hline IHD & $121(25.5)$ & $52(31.3)$ & $127(29.3)$ & \multirow[t]{2}{*}{0.25} \\
\hline CRRT & $354(74.5)$ & $114(68.7)$ & $307(70.7)$ & \\
\hline CRRT duration (days), median (IQR) & $3.9(1.5-7.7)$ & $5.8(3.6-9.4)$ & $5.9(2.8-9.5)$ & $<0.001$ \\
\hline UF $^{\mathrm{NET}}$ volume during CRRT $(\mathrm{L})$, median (IQR) & $3.4(0.9-10.2)$ & $11.6(5.4-19.2)$ & $16.2(7.5-28.4)$ & $<0.001$ \\
\hline IHD duration (days), median (IQR) & $2(5-9)$ & $7(3-13)$ & $4(2-8)$ & 0.004 \\
\hline $\mathrm{UF}^{\mathrm{NET}}$ volume during IHD $(\mathrm{L})$, median (IQR) & $5.5(2.2-13.5)$ & $12.6(4.4-19.7)$ & $9.2(4-17.2)$ & $<0.001$ \\
\hline Both CRRT and IHD duration (days), median (IQR) & $14.7(9.7-22.9)$ & $15.2(9.2-21.9)$ & $10.7(6.9-18.4)$ & $<0.001$ \\
\hline UF $^{\text {NET }}$ volume during CRRT and IHD (L), median (IQR) & $19.5(9.5-33.9)$ & $27.9(18.5-42.1)$ & $26.6(17.8-46.1)$ & $<0.001$ \\
\hline Hospital length of stay (days), median (IQR) & $32(17-54)$ & $37.5(23-65)$ & $37(23-61)$ & $<0.001$ \\
\hline Hospital mortality & $272(57.3)$ & $70(42.2)$ & $187(43.1)$ & $<0.001$ \\
\hline 1-year mortality & $331(69.7)$ & $100(60.2)$ & $258(59.4)$ & 0.003 \\
\hline Renal recovery at 1 year ${ }^{b}$ & $119(25.1)$ & $48(28.9)$ & $138(31.8)$ & 0.078 \\
\hline Renal recovery at 1 year in survivors ${ }^{b}$ & $119(82.6)$ & $48(72.7)$ & $138(78.4)$ & 0.25 \\
\hline
\end{tabular}

Data presented as $n$ (\%) unless stated otherwise

$R R T$ renal replacement therapy, ICU intensive care unit, $I Q R$ interquartile range, $F B$ fluid balance, $U F^{N E T}$ net ultrafiltration, $M A P$ mean arterial pressure, SD standard deviation, NE norepinephrine equivalents, IHD intermittent hemodialysis, CRRT continuous renal replacement therapy

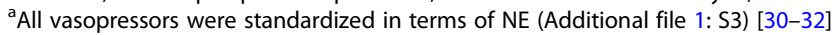

${ }^{\mathrm{b}}$ Renal recovery was defined as alive and independent of RRT at 1 year

using tertile cutoff values (AOR 0.61, 95\% CI 0.39-0.97, $p=0.037$; Table 5). The quantitative bias analysis indicated that our results would be robust unless an unmeasured confounder was at least twice as prevalent among patients who received high-intensity $\mathrm{UF}^{\mathrm{NET}}$ as among those with low-intensity $\mathrm{UF}^{\mathrm{NET}}$ (Additional file 1: Figure S4). The unmeasured confounder should have an OR $<0.7$ (i.e., reduced risk of death by more than $30 \%$ ) to mask a null association between high-intensity $\mathrm{UF}^{\mathrm{NET}}$ and risk-adjusted mortality (Additional file 1: S6 and Figure S4).

\section{Subgroup analyses}

High-intensity $\mathrm{UF}^{\mathrm{NET}}$ was associated with a trend toward lower mortality among patients with $>20 \%$ FO
(AOR 0.52, 95\% CI 0.26-1.05, $p=0.07$; Table 5). For patients receiving CRRT, UF ${ }^{\mathrm{NET}}$ intensity $>1.0 \mathrm{ml} / \mathrm{kg} / \mathrm{h}$ compared with $\mathrm{UF}^{\mathrm{NET}}$ intensity $<0.5 \mathrm{ml} / \mathrm{kg} / \mathrm{h}$ was associated with lower odds of death (AOR 0.41, 95\% CI $0.24-0.71, p=0.0013$ ).

\section{Discussion}

We found that $\mathrm{UF}^{\mathrm{NET}}$ intensity $>25 \mathrm{ml} / \mathrm{kg} /$ day, compared with $<20 \mathrm{ml} / \mathrm{kg} /$ day, was independently associated with lower risk-adjusted 1-year mortality in critically ill patients with FO. Using Gray's model, this survival benefit was greater early on after ICU admission and persisted up to 39 days. In the propensity-matched analysis, $\mathrm{UF}^{\mathrm{NET}}>25 \mathrm{ml} / \mathrm{kg} /$ day, compared with $\leq 25 \mathrm{ml} / \mathrm{kg} /$ day, 

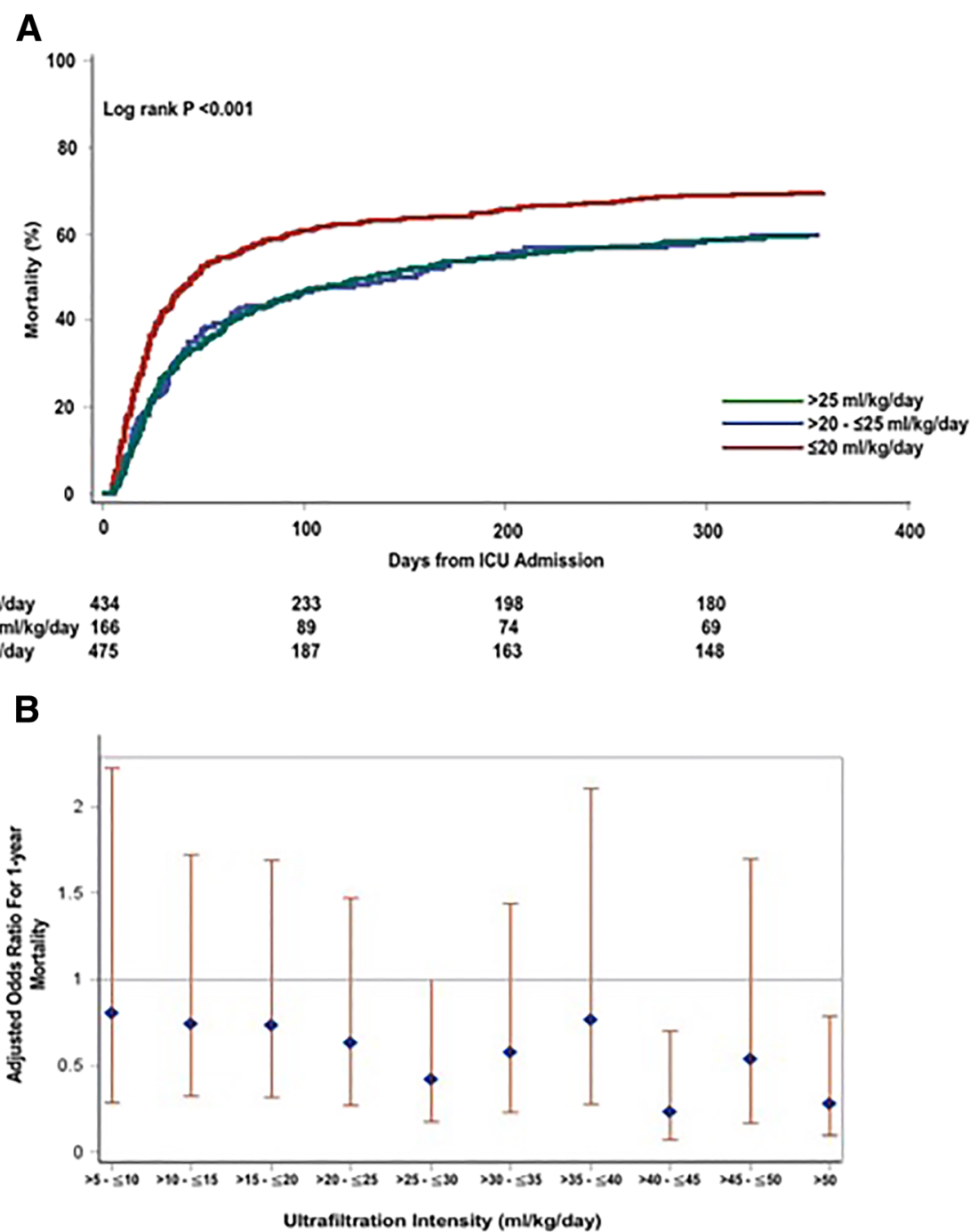

Fig. 1 a. Association between net ultrafiltration intensity and time to mortality. Kaplan-Meier failure plots by UF ${ }^{\mathrm{NET}}$ intensity for probability of death over 1 year from ICU admission in overall cohort $(n=1075)$. Red line, low-intensity UF ${ }^{\mathrm{NET}}(\leq 20 \mathrm{ml} / \mathrm{kg} /$ day); blue line, moderate-intensity $\mathrm{UF}^{\mathrm{NET}}$ (> 20 to $\leq 25 \mathrm{ml} / \mathrm{kg} /$ day); green line, high-intensity UF ${ }^{\mathrm{NET}}$ (> $25 \mathrm{ml} / \mathrm{kg} /$ day). Probability of death highest in low-intensity compared with moderate and high-intensity UF ${ }^{\mathrm{NET}}$ groups (log-rank $p<0.001$ ). b. Association between net ultrafiltration intensity and risk-adjusted 1-year mortality Shown are adjusted odds ratio with $95 \% \mathrm{Cl}$ for association between UF ${ }^{\mathrm{NET}}$ intensity and mortality. Increasing UF ${ }^{\mathrm{NET}}$ intensity associated with trend toward lower mortality. Odds ratios adjusted for differences in age, sex, race, BMl, history of liver disease and sequela from liver disease, admission for liver transplantation, admission for surgery, baseline glomerular filtration rate, Acute Physiology and Chronic Health Evaluation III score, presence of sepsis, use of mechanical ventilation, percentage of cumulative fluid overload before initiation of RRT, oliguria before initiation of RRT, time to initiation of RRT from ICU admission, MAP on first day of RRT initiation, cumulative vasopressor dose and cumulative fluid balance during RRT, first RRT modality and duration of RRT. ICU intensive care unit

Table 3 Association between UF ${ }^{\mathrm{NET}}$ intensity and 1-year risk-adjusted mortality

\begin{tabular}{|c|c|c|c|c|}
\hline Covariates & Unadjusted odds ratio $(95 \% \mathrm{Cl})$ & $p$ value & Adjusted $^{a}$ odds ratio $(95 \% \mathrm{Cl})$ & $p$ value \\
\hline Moderate vs low-intensity UF NET (reference) & $0.65(0.42-0.94)$ & 0.024 & $0.81(0.48-1.35)$ & 0.41 \\
\hline High vs low-intensity UF ${ }^{\mathrm{NET}}$ (reference) & $0.64(0.49-0.85)$ & 0.002 & $0.61(0.41-0.93)$ & 0.02 \\
\hline
\end{tabular}

$U F^{N E T}$ net ultrafiltration, $C l$ confidence interval, FO fluid overload, RRT renal replacement therapy, ICU intensive care unit

${ }^{a}$ Adjusted for age, sex, race, body mass index, history of liver disease and sequela from liver disease, admission for liver transplantation, admission for surgery, baseline glomerular filtration rate, Acute Physiology and Chronic Health Evaluation III score, presence of sepsis, use of mechanical ventilation, percentage of FO before initiation of RRT, oliguria before initiation of RRT, time to initiation of RRT from ICU admission, mean arterial pressure on first day of RRT initiation, cumulative vasopressor dose and cumulative fluid balance during RRT, first RRT modality and duration of RRT 
Table 4 Association between intensity of net ultrafiltration and time to mortality from Gray's model

\begin{tabular}{lllllll}
\hline Characteristic & \multicolumn{4}{l}{ Adjusted hazard ratio $(95 \% \mathrm{Cl})$ by time interval } & & \\
\cline { 2 - 5 } & $5-15$ days & $15-23$ days & $23-39$ days & $39-91$ days & $91-365$ days \\
\hline High vs low UF & $0.50(0.35-0.71)$ & $0.62(0.46-0.82)$ & $0.73(0.55-0.97)$ & $0.76(0.56-1.04)$ & $1.02(0.71-1.47)$ & $<0.001$ \\
High vs moderate UF & $0.53(0.33-0.86)$ & $0.69(0.46-1.02)$ & $0.75(0.52-1.09)$ & $0.77(0.518-1.142)$ & $1.16(0.72-1.85)$ & 0.039 \\
Moderate vs low UF & $0.98(0.62-1.57)$ & $0.87(0.59-1.27)$ & $0.996(0.69-1.43)$ & $1.01(0.69-1.47)$ & $0.844(0.53-1.34)$ & 0.91 \\
\hline
\end{tabular}

Shown are adjusted hazard ratios estimated from Gray's model for association between intensity of UF ${ }^{\text {NET }}$ and mortality for each time interval. Models included five time intervals and four time nodes with the default timing of nodes chosen by the statistical program based on the number of observations within each time interval. Hazard ratio $<1$ suggests that $\mathrm{UF}^{\mathrm{NET}}$ intensity is associated with lower mortality, and hazard ratio $>1$ suggests $\mathrm{UF}^{\mathrm{NET}}$ intensity is associated with higher mortality. $p$ values reported are for the ranges of hazard ratios from the model

$\mathrm{Cl}$ confidence interval, UF ${ }^{N E T}$ net ultrafiltration, FO fluid overload, RRT renal replacement therapy, ICU intensive care unit

adjusted for age, sex, race, body mass index, history of liver disease and sequela from liver disease, admission for liver transplantation, admission for surgery, baseline glomerular filtration rate, Acute Physiology and Chronic Health Evaluation III score, presence of sepsis, use of mechanical ventilation, percentage of FO before initiation of RRT, oliguria before initiation of RRT, time to initiation of RRT from ICU admission, mean arterial pressure on first day of RRT initiation, cumulative vasopressor dose and cumulative fluid balance during RRT, first RRT modality and duration of RRT

was also associated with lower risk of death. To our knowledge, this is the first study in the literature examining the association between $\mathrm{UF}^{\mathrm{NET}}$ intensity and long-term mortality.

Our finding is somewhat analogous to the association between intensity of solute control and mortality in critically ill patients receiving RRT in which a threshold intensity of at least $20-25 \mathrm{ml} / \mathrm{kg} / \mathrm{h}$ of effluent dosing in CRRT or KT/V of 1.2-1.4 per session in patients receiving IHD is associated with improved survival [26, 27]. However, in contrast to studies on solute control, the optimal "dosing" for UF ${ }^{\mathrm{NET}}$ in critically ill patients with fluid overload is unclear. In our study, we first explored whether there was an association between $\mathrm{UF}^{\mathrm{NET}}$ dose and mortality, and then aimed to determine the overall "average dose" that is associated with a long-term mortality benefit. It is important to note that our finding does not suggest that $\mathrm{UF}^{\mathrm{NET}}$ should be dosed $>25 \mathrm{ml} /$ $\mathrm{kg} /$ day throughout the duration of fluid removal. Day-to-day dosing may vary in patients depending on the severity of fluid overload, patient tolerability and hemodynamics.

In our study only $40 \%$ of patients received intensive $\mathrm{UF}^{\mathrm{NET}}$, whereas $44 \%$ of patients received less intensive $\mathrm{UF}^{\mathrm{NET}}$ that has implications for care. Unlike a prescription for solute clearance, the concept of a minimum or adequate "dose" for volume clearance is not usually considered in clinical practice. Although patients who received less intensive $\mathrm{UF}^{\mathrm{NET}}$ were hemodynamically unstable in our study, our findings persisted after accounting for hemodynamics, vasopressor dose and severity of illness, suggesting that less intensive $U^{N E T}$ per

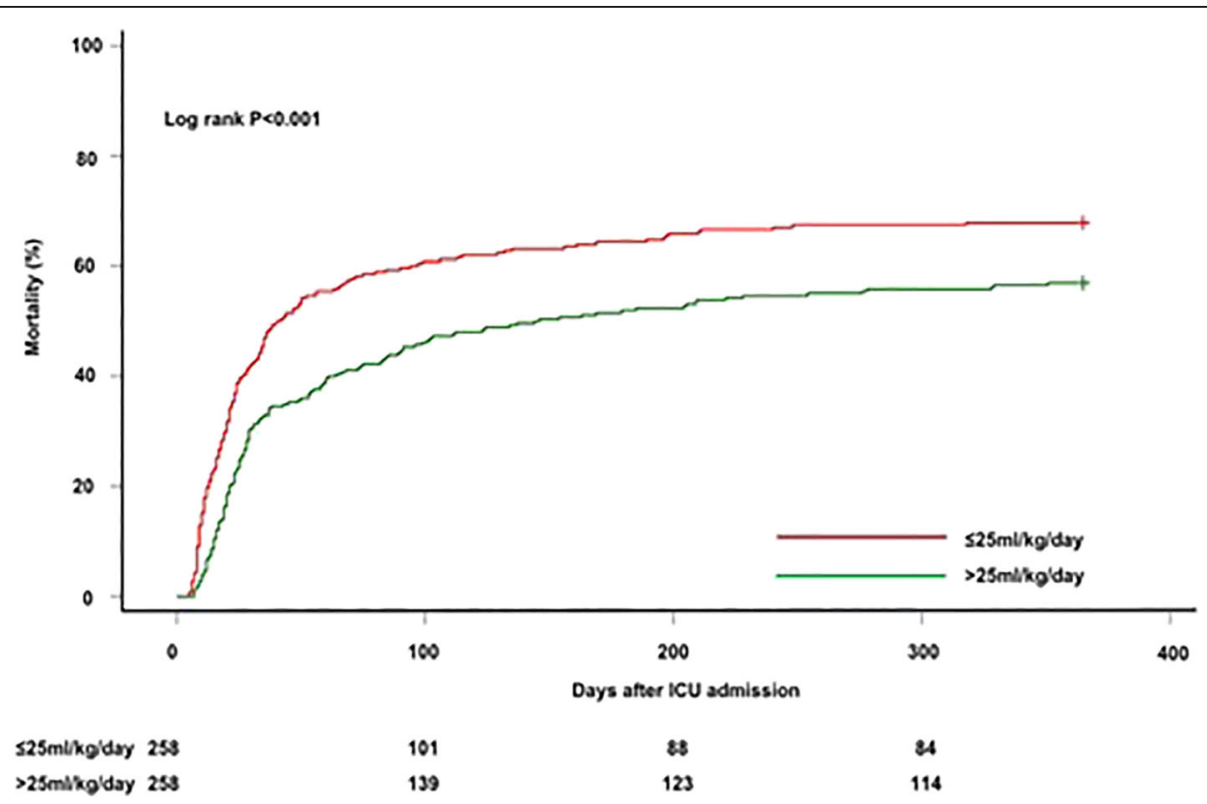

Fig. 2 Association between net ultrafiltration intensity and time to mortality in propensity-matched cohort. Kaplan-Meier failure plots by UF ${ }^{\text {NET }}$ for probability of death over 1 year from ICU admission among patients with UF ${ }^{\mathrm{NET}} \leq 25 \mathrm{ml} / \mathrm{kg} /$ day $(n=258)$ compared with propensity-matched patients with UF ${ }^{\mathrm{NET}}>25 \mathrm{ml} / \mathrm{kg} /$ day $(n=258)$. Red line, UF ${ }^{\mathrm{NET}} \leq 25 \mathrm{ml} / \mathrm{kg} / \mathrm{day}$; green line, $\mathrm{UF}^{\mathrm{NET}}>25 \mathrm{ml} / \mathrm{kg} /$ day. Probability of death lower among patients who received UF ${ }^{\mathrm{NET}}>25 \mathrm{ml} / \mathrm{kg} /$ day compared with $\mathrm{UF}^{\mathrm{NET}} \leq 25 \mathrm{ml} / \mathrm{kg} /$ day (log-rank $p<0.001$ ). ICU intensive care unit 
Table 5 Sensitivity and subgroup analyses of net ultrafiltration intensity and mortality

\begin{tabular}{|c|c|c|c|}
\hline Characteristic & Net ultrafiltration intensity & Adjusted odds ratio $(95 \% \mathrm{Cl})^{\mathrm{a}}$ & $p$ value \\
\hline \multicolumn{4}{|l|}{ Sensitivity analysis } \\
\hline \multirow[t]{2}{*}{ UF $^{N E T}$ up to $72 \mathrm{~h}$ after RRT initiation ${ }^{\mathrm{b}}$} & High vs low & $0.56(0.35-0.88)$ & 0.013 \\
\hline & Moderate vs low & $1.10(0.58-2.11)$ & 0.76 \\
\hline \multirow[t]{2}{*}{ Alternative UF ${ }^{N E T}$ threshold ${ }^{C}$} & High vs low & $0.63(0.41-0.97)$ & 0.038 \\
\hline & Moderate vs low & $0.91(0.53-1.58)$ & 0.74 \\
\hline \multirow[t]{2}{*}{ Alternative UF ${ }^{N E T}$ threshold $^{d}$} & High vs low & $0.58(0.34-0.99)$ & 0.044 \\
\hline & Moderate vs low & $0.66(0.43-1.01)$ & 0.053 \\
\hline \multirow[t]{2}{*}{ Alternative UF ${ }^{N E T}$ threshold } & High vs low & $0.61(0.39-0.97)$ & 0.0371 \\
\hline & Moderate vs low & $0.69(0.45-1.07)$ & 0.096 \\
\hline \multicolumn{4}{|l|}{ Subgroup analysis } \\
\hline \multirow{2}{*}{$\begin{array}{l}\text { UF } \\
\text { cumulative } F B>20 \% \text { before } \text { RRT }^{f}\end{array}$} & High vs low & $0.52(0.26-1.05)$ & 0.07 \\
\hline & Moderate vs low & $0.74(0.29-1.84)$ & 0.51 \\
\hline \multirow{2}{*}{$\begin{array}{l}\text { Alternative UF }{ }^{N E T} \text { threshold among subgroup } \\
\text { of patients who only received CRRT }\end{array}$} & High vs low & $0.41(0.24-0.71)$ & 0.0013 \\
\hline & Moderate vs low & $0.68(0.39-1.18)$ & 0.17 \\
\hline \multicolumn{4}{|c|}{$\begin{array}{l}\text { CI confidence interval, UF }{ }^{N E T} \text { net ultrafiltration, RRT renal replacement therapy, FB fluid balance, CRRT continuous renal replacement therapy, FO fluid overload, ICU } \\
\text { intensive care unit } \\
\text { aAdjusted for differences in age, sex, race, body mass index, history of liver disease and sequela from liver disease, admission for liver transplantation, admission } \\
\text { for surgery, baseline glomerular filtration rate, Acute Physiology and Chronic Health Evaluation III score, presence of sepsis, use of mechanical ventilation, } \\
\text { percentage of FO before initiation of RRT, oliguria before initiation of RRT, time to initiation of RRT from ICU admission, mean arterial pressure on first day of RRT } \\
\text { initiation, cumulative vasopressor dose and fluid balance during RRT, first RRT modality and duration of RRT } \\
\text { bUF }\end{array}$} \\
\hline \multicolumn{4}{|c|}{ 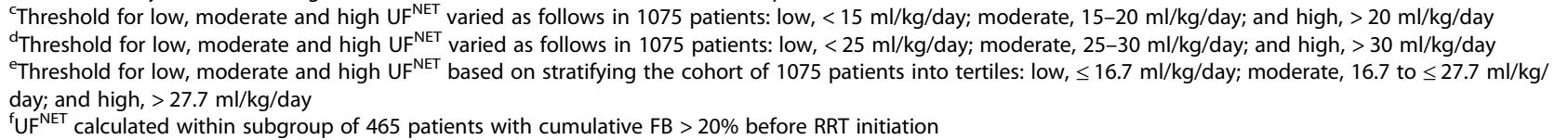 } \\
\hline \multicolumn{4}{|c|}{$\begin{array}{l}\text { IThreshold for low, moderate and high UF }{ }^{\mathrm{NET}} \text { varied among subgroup of } 487 \text { patients who only received CRRT as follows: low, }<0.5 \mathrm{ml} / \mathrm{kg} / \mathrm{h} ; \mathrm{moderate}, 0.5-1.0 \mathrm{ml} / \mathrm{kg} / \mathrm{h} \text {; } \\
\text { and high, }>1 \mathrm{ml} / \mathrm{kg} / \mathrm{h}\end{array}$} \\
\hline
\end{tabular}

se might be associated with mortality. These findings may suggest that failure to tolerate $\mathrm{UF}^{\mathrm{NET}}>25 \mathrm{ml} / \mathrm{kg} /$ day may portend a poor prognosis and, conversely, tolerating $\mathrm{UF}^{\mathrm{NET}}>25 \mathrm{ml} / \mathrm{kg} /$ day may be a predictor of recovery and lower mortality in critically ill patients with fluid overload.

Our study addresses an important knowledge gap not addressed by prior studies. While numerous studies have documented an association between the severity of FO and incremental risk of death [3, 4], none examined the $\mathrm{UF}^{\mathrm{NET}}$ intensity-mortality relationship. Using the Program to Improve Care in Acute Renal Disease (PICARD) study, Bouchard et al. [4] found that patients in whom FO was corrected during RRT had lower mortality than those who remained fluid overloaded despite RRT. Using the Randomized Evaluation of Normal versus Augmented Level of Renal Replacement Therapy (RENAL RRT) cohort, Bellomo et al. [2] found that a negative fluid balance during RRT was associated with a mortality benefit. However, we asked a different question: does $\mathrm{UF}^{\mathrm{NET}}$ intensity and a threshold "dose" of UF ${ }^{\mathrm{NET}}$ matter in the treatment of FO independent of fluid balance?

There may be several biologic explanations for the association between $\mathrm{UF}^{\mathrm{NET}}$ intensity and outcome. First, intensive $\mathrm{UF}^{\mathrm{NET}}$ may reduce prolonged exposure to $\mathrm{FO}$ and modify host response, and could reduce the incidence of subsequent organ dysfunction [28]. Second, the salutary effects of intensive $\mathrm{UF}^{\mathrm{NET}}$ may be mediated through unknown marker clearance independent of fluid balance since the association persisted despite controlling for cumulative fluid balance. Third, clinicians who decide to initiate intensive $\mathrm{UF}^{\mathrm{NET}}$ may select for a unique group of patients to monitor and carefully titrate fluid removal. Fourth, clinicians and nurses may also have a broad variation in how they prescribe and/or practice $\mathrm{UF}^{\mathrm{NET}}$ in the real world, which may be associated with differences in outcomes [29].

The strengths of our study was that it was robust to three different methods of sensitivity analysis. We accounted for confounding due to severity of illness, hemodynamics, vasopressor dose and cumulative fluid balance before and during RRT. Using Gray's model, we found that high-intensity UF ${ }^{\mathrm{NET}}$ was associated with survival only up to 39 days after ICU admission. This finding is in contrast with the logistic model and propensity-matched analyses, which showed mortality benefit up to 1 year. This discordant finding is due to the differences in the models that were used. In Gray's model, the number of events 
between high-intensity and low-intensity $\mathrm{UF}^{\mathrm{NET}}$ groups was not different within the time interval of 39-365 days. Using the logistic regression model, however, a lower odds of cumulative deaths occurred by 1 year in the high->in$>$ intensity $\mathrm{UF}^{\mathrm{NET}}$ group compared with the <?A3B2 thy$\mathrm{c}=$ low-intensity $\mathrm{UF}^{\mathrm{NET}}$ group.

Our study is not without limitations. First, given the observational nature, it is not possible to make causal inferences between UF ${ }^{\mathrm{NET}}$ intensity and outcomes. Second, we do not know precisely whether a $\mathrm{UF}^{\mathrm{NET}}$ threshold $>25 \mathrm{ml} / \mathrm{kg} /$ day is associated with better outcomes, although our findings were robust to several sensitivity analyses. Third, our single-center study may not be generalizable to other ICU populations. Nevertheless, our study included patients typical of an academic medical center ICU population. Fourth, we were unable to distinguish whether patients received low-intensity UF $^{\text {NET }}$ due to low prescription, failure to remove fluid (e.g., circuit downtime, trip to operating room, etc.) or other variations in practice with respect to fluid removal. Fifth, although the sensitivity analysis indicated that any unmeasured confounder would need to be highly prevalent and have an $\mathrm{OR}<0.7$ to mask a null association, it is possible that there may be more than one residual confounder and that it may not be a binary variable.

\section{Conclusion}

In summary, among critically ill patients with $\geq 5 \%$ FO receiving RRT, our study found that $\mathrm{UF}^{\mathrm{NET}}$ intensity > $25 \mathrm{ml} / \mathrm{kg} /$ day is associated with lower risk-adjusted 1-year mortality compared with $<20 \mathrm{ml} / \mathrm{kg} /$ day. Whether this association between $\mathrm{UF}^{\mathrm{NET}}$ intensity $>25 \mathrm{ml} / \mathrm{kg} /$ day and lower mortality risk is just a marker for recovery or a mediator needs to be refuted or confirmed in future prospective randomized controlled trials.

\section{Additional file}

Additional file 1: S1. Study population. S2. Determination of cumulative fluid balance. S3. Vasopressor standardization to norepinephrine equivalents. S4. Gray's survival model. S5. Propensity score estimation and matching. S6. Quantitative bias sensitivity analysis of potential impact of an unmeasured confounder. Figure S1. Study population and analysis cohort. Figure S2. Association between intensity of net ultrafiltration and crude hospital mortality. Figure S3. Association between net ultrafiltration intensity and time to mortality using Gray's model. Figure S4. Quantitative bias sensitivity analysis to assess the impact of an unmeasured confounder on mortality. Table S1. Cumulative fluid balance, mean arterial pressure and vasopressor dose for entire duration of RRT. Table S2. Association between net ultrafiltration intensity and 1-year risk-adjusted mortality. Table S3. Association between net ultrafiltration intensity and 1-year risk-adjusted mortality using net ulftrafiltration as a continuous variable. Table S4. Baseline characteristics by net ultrafiltration intensity after propensity matching (PDF $2022 \mathrm{~kb}$ )

\section{Abbreviations}

AHR: Adjusted hazard ratio; AKl: Acute kidney injury; AOR: Adjusted odds ratio; APACHE: Acute Physiology and Chronic Health Evaluation; CRRT: Continuous renal replacement therapy; $\mathrm{CWH}$ : Continuous venovenous hemofiltration;
CWHD: Continuous venovenous hemodialysis; CWHDF: Continuous venovenous hemodiafiltration; FO: Fluid overload; IHD: Intermittent hemodialysis; MAP: Mean arterial pressure; RRT: Renal replacement therapy; SCUF: Slow continuous ultrafiltration; UF ${ }^{\mathrm{NET}}$ : Net ultrafiltration

\section{Availability of data and materials}

The datasets generated and/or analyzed during the current study are not publicly available as they belong to the University of Pittsburgh and University of Pittsburgh Medical Center but are available from the corresponding author on reasonable request.

\section{Authors' contributions}

RM, VB, SJK and PP had full access to all of the data in the study and take responsibility for the integrity of the data and the accuracy of the data analysis. RM and VB were responsible for study concept and design. JAK and GC were responsible for acquisition of data. SJK, C-CHC, PP, RM, VB, GC, PMP, $\mathrm{RB}$ and JAK analyzed and interpreted data. RM and VB drafted the manuscript. JAK, RB, PMP, GC, VB, RM, C-CHC, SJK and PP critically revised the manuscript for important intellectual content. VB and SJK performed statistical analysis. VB, RM and PP provided administrative, technical or material support. RM supervised the study. All authors read and approved the final manuscript.

\section{Ethics approval and consent to participate}

This project was approved by the University of Pittsburgh Institutional Review Board.

\section{Consent for publication}

Not applicable.

\section{Competing interests}

The authors declare that they have no competing interests.

\section{Publisher's Note}

Springer Nature remains neutral with regard to jurisdictional claims in published maps and institutional affiliations.

\section{Author details}

${ }^{1}$ Department of Critical Care Medicine, The Center for Critical Care Nephrology, CRISMA, University of Pittsburgh School of Medicine, Pittsburgh, PA, USA. ${ }^{2}$ Department of Critical Care Medicine, The Clinical Research, Investigation, and Systems Modeling of Acute IIIness (CRISMA) Center, University of Pittsburgh School of Medicine, Pittsburgh, PA, USA. ${ }^{3}$ Department of Medicine, University of Pittsburgh School of Medicine, Pittsburgh, PA, USA. ${ }^{4}$ Department of Biostatistics, Graduate School of Public Health, University of Pittsburgh, Pittsburgh, PA, USA. ${ }^{5}$ Department of Intensive Care Medicine, The University of Melbourne, Austin Hospital, Heidelberg, VIC, Australia. ${ }^{6}$ Renal Section, Veterans Affairs Pittsburgh Healthcare System, Pittsburgh, PA, USA. ${ }^{7}$ Critical Care Medicine, and Clinical \& Translational Science, University of Pittsburgh, Suite 220, Room 206, 3347 Forbes Avenue, Pittsburgh, PA 15261, USA.

Received: 28 May 2018 Accepted: 16 August 2018 Published online: 24 September 2018

\section{References}

1. Balakumar V, Murugan R, Sileanu FE, Palevsky P, Clermont G, Kellum JA. Both positive and negative fluid balance may be associated with reduced long-term survival in the critically ill. Crit Care Med. 2017;45(8):e749-57.

2. Bellomo R, Cass A, Cole L, Finfer S, Gallagher M, Lee J, Lo S, McArthur C, McGuiness $S$, et al. An observational study fluid balance and patient outcomes in the randomized evaluation of normal vs. augmented level of replacement therapy trial. Crit Care Med. 2012;40(6):1753-60.

3. Vaara ST, Korhonen AM, Kaukonen KM, Nisula S, Inkinen O, Hoppu S, Laurila JJ, Mildh L, Reinikainen M, Lund V, et al. Fluid overload is associated with an increased risk for 90-day mortality in critically ill patients with renal replacement therapy: data from the prospective FINNAKI study. Crit Care. 2012;16(5):R197.

4. Bouchard J, Soroko SB, Chertow GM, Himmelfarb J, Ikizler TA, Paganini EP, Mehta RL, Program to Improve Care in Acute Renal Disease Study Group. Fluid accumulation, survival and recovery of kidney function in critically ill patients with acute kidney injury. Kidney Int. 2009;76(4):422-7. 
5. KDIGO. Clinical practice guideline for acute kidney injury. Kidney Int Suppl. 2012;2:1-138.

6. Rosner MH, Ostermann M, Murugan R, Prowle JR, Ronco C, Kellum JA, Mythen MG, Shaw AD, ADQI XII Investigators Group. Indications and management of mechanical fluid removal in critical illness. Br J Anaesth. 2014;113(5):764-71.

7. Alwall N. On the artificial kidney; apparatus for dialysis of the blood in vivo. Acta Med Scand. 1947;128(4):317-25.

8. Flythe JE, Curhan GC, Brunelli SM. Disentangling the ultrafiltration ratemortality association: the respective roles of session length and weight gain. Clin J Am Soc Nephrol. 2013;8(7):1151-61.

9. Davies SJ, Brown EA, Reigel W, Clutterbuck E, Heimburger O, Diaz NV, Mellote GJ, Perez-Contreras J, Scanziani R, D'Auzac C, et al. What is the link between poor ultrafiltration and increased mortality in anuric patients on automated peritoneal dialysis? Analysis of data from EAPOS. Perit Dial Int. 2006;26(4):458-65.

10. Burton JO, Jefferies HJ, Selby NM, Mclntyre CW. Hemodialysis-induced repetitive myocardial injury results in global and segmental reduction in systolic cardiac function. Clin J Am Soc Nephrol. 2009;4(12):1925-31.

11. Silversides JA, Pinto R, Kuint R, Wald R, Hladunewich MA, Lapinsky SE, Adhikari NK. Fluid balance, intradialytic hypotension, and outcomes in critically ill patients undergoing renal replacement therapy: a cohort study. Crit Care. 2014;18(6):624.

12. Flythe JE, Kimmel SE, Brunelli SM. Rapid fluid removal during dialysis is associated with cardiovascular morbidity and mortality. Kidney Int. 2011; 79(2):250-7.

13. Movilli E, Gaggia P, Zubani R, Camerini C, Vizzardi V, Parrinello G, Savoldi S, Fischer MS, Londrino F, Cancarini G. Association between high ultrafiltration rates and mortality in uraemic patients on regular haemodialysis. A 5-year prospective observational multicentre study. Nephrol Dial Transplant. 2007; 22(12):3547-52.

14. Saran R, Bragg-Gresham JL, Levin NW, Twardowski ZJ, Wizemann V, Saito A, Kimata N, Gillespie BW, Combe C, Bommer J, et al. Longer treatment time and slower ultrafiltration in hemodialysis: associations with reduced mortality in the DOPPS. Kidney Int. 2006;69(7):1222-8.

15. Rewa OG, Villeneuve PM, Lachance P, Eurich DT, Stelfox HT, Gibney RTN, Hartling L, Featherstone R, Bagshaw SM. Quality indicators of continuous renal replacement therapy (CRRT) care in critically ill patients: a systematic review. Intensive Care Med. 2017;43(6):750-63.

16. Flythe JE, Assimon MM, Wenger JB, Wang L. Ultrafiltration rates and the quality incentive program: proposed measure definitions and their potential dialysis facility implications. Clin J Am Soc Nephrol. 2016;11(8):1422-33.

17. Kellum JA, Murugan R. Effects of non-severe acute kidney injury on clinical outcomes in critically ill patients. Crit Care. 2016;20(1):159.

18. Liang KV, Sileanu FE, Clermont G, Murugan R, Pike F, Palevsky PM, Kellum JA. Modality of RRT and recovery of kidney function after AKI in patients surviving to hospital discharge. Clin J Am Soc Nephrol. 2016;11(1):30-8.

19. Neri M, Villa G, Garzotto F, Bagshaw S, Bellomo R, Cerda J, Ferrari F, Guggia $\mathrm{S}$, Joannidis $\mathrm{M}$, Kellum J, et al. Nomenclature for renal replacement therapy in acute kidney injury: basic principles. Crit Care. 2016;20(1):318.

20. Hill ME, Rosenwaike I. Social Security Administration's Death Master File: the completeness of death reporting at older ages. Soc Sec Bull. 2001;64:45.

21. Saran R, Li Y, Robinson B, Ayanian J, Balkrishnan R, Bragg-Gresham J, Chen J, Cope E, Gipson D, He K. US Renal Data System 2014 annual data report: epidemiology of kidney disease in the United States. Am J Kidney Dis. 2015; 65(6 Suppl 1):A7.

22. Kasal J, Jovanovic Z, Clermont G, Weissfeld LA, Kaplan V, Watson RS, Angus DC. Comparison of cox and Gray's survival models in severe sepsis. Crit Care Med. 2004;32(3):700-7.

23. Valenta Z, Weissfeld L. Estimation of the survival function for Gray's piecewise-constant time-varying coefficients model. Stat Med. 2002;21(5): 717-27.

24. Lash T, Fox MP, Fink AK. Applying quantitative bias analysis to epidemiologic data. New York: Springer-Verlag; 2009.

25. Lin DY, Psaty BM, Kronmal RA. Assessing the sensitivity of regression results to unmeasured confounders in observational studies. Biometrics. 1998;54(3): 948-63.

26. Network VNARFT, Palevsky PM, Zhang JH, O'Connor TZ, Chertow GM, Crowley ST, Choudhury D, Finkel K, Kellum JA, Paganini E, et al. Intensity of renal support in critically ill patients with acute kidney injury. N Engl J Med. 2008:359(1):7-20.
27. Bellomo R, Cass A, Cole L, Finfer S, Gallagher M, Lo S, McArthur C, McGuinness S, Myburgh J, et al. Intensity of continuous renal-replacement therapy in critically ill patients. N Engl J Med. 2009;361 (17):1627-38.

28. O'Connor ME, Prowle JR. Fluid overload. Crit Care Clin. 2015;31(4):803-21.

29. O'Connor ME, Jones SL, Glassford NJ, Bellomo R, Prowle JR. Defining fluid removal in the intensive care unit: a national and international survey of critical care practice. J Intensive Care Soc. 2017;18(4):282-8.

30. Khanna A, English SW, Wang XS, Ham K, Tumlin J, Szerlip H, Busse LW, Altaweel L, Albertson TE, Mackey C, et al. Angiotensin II for the treatment of vasodilatory shock. N Engl J Med. 2017;377(5):419-30.

31. Russell JA, Walley KR, Singer J, Gordon AC, Hebert PC, Cooper DJ, Holmes CL, Mehta S, Granton JT, Storms MM, et al. Vasopressin versus norepinephrine infusion in patients with septic shock. N Engl J Med. 2008; 358(9):877-87.

32. Vincent JL, Moreno R, Takala J, Willatts S, De Mendonca A, Bruining H, Reinhart CK, Suter PM, Thijs LG. The SOFA (Sepsis-related Organ Failure Assessment) score to describe organ dysfunction/failure. On behalf of the Working Group on Sepsis-Related Problems of the European Society of Intensive Care Medicine. Intensive Care Med. 1996;22(7):707-10.

Ready to submit your research? Choose BMC and benefit from:

- fast, convenient online submission

- thorough peer review by experienced researchers in your field

- rapid publication on acceptance

- support for research data, including large and complex data types

- gold Open Access which fosters wider collaboration and increased citations

- maximum visibility for your research: over $100 \mathrm{M}$ website views per year

At $\mathrm{BMC}$, research is always in progress.

Learn more biomedcentral.com/submissions 